\title{
Reduced Brain Activation in Response to Social Cognition Tasks in Autism Spectrum Disorder with and without Depression
}

\author{
Toshiyuki Ohtani ${ }^{1-3}$ \\ Koji Matsuo ${ }^{4}$ \\ Chihiro Sutoh ${ }^{5}$ \\ Fumiyo Oshima ${ }^{2,3}$ \\ Yoshiyuki Hirano (D) ${ }^{2,3}$ \\ Akio Wakabayashi ${ }^{6}$ \\ Eiji Shimizu ${ }^{2,3,5}$
}

'Safety and Health Organization, Chiba University, Chiba, Japan; ${ }^{2}$ Research

Center for Child Mental Development, Chiba University, Chiba, Japan; ${ }^{3}$ United Graduate School of Child Development, Osaka University, Kanazawa University, Hamamatsu University School of Medicine, Chiba University and University of Fukui, Suita, Japan; ${ }^{4}$ Department of Psychiatry, Faculty of Medicine, Saitama Medical University, Saitama, Japan; ${ }^{5}$ Department of Cognitive Behavioral Physiology, Graduate School of Medicine and School of Medicine, Chiba University, Chiba University, Chiba, Japan; ${ }^{6}$ Department of Psychology, Graduate School of Humanities, Chiba University, Chiba, Japan
Correspondence: Toshiyuki Ohtani Safety and Health Organization, Chiba University, I-33 Yayoicho, Inage-ku, Chiba, 263-8522, Japan

$\mathrm{Tel} / \mathrm{Fax}+81432902216$

Email otanit@Chiba-u.jp
Purpose: In clinical settings, diagnosing comorbid depression in individuals with autism spectrum disorder (ASD) is often difficult. Neuroimaging studies have reported reduced activation of frontal and temporal regions during emotional face recognition task (EFRT) in ASD and depression. However, to the best of our knowledge, no study has examined differences in frontotemporal activation during EFRT between ASD with and without comorbid depression. We aimed to compare the frontotemporal hemodynamic responses to the EFRT in ASD with and without depression and to find clues to help in discriminating the characteristics between them.

Patients and Methods: In 24 drug-naïve young adults with ASD (12 with depression [ASD-Dep(+)] and 12 without depression [ASD-Dep(-)]) and 12 with typical development (TD), frontotemporal hemodynamic responses during an EFRT were measured using functional near-infrared spectroscopy (fNIRS).

Results: The ASD groups showed reduced activation during EFRT than the TD group in the right ventrolateral prefrontal cortex (VLPFC). Moreover, the ASD-Dep $(+)$ group showed reduced activation during EFRT than the ASD-Dep(-) group in the right anterior temporal cortex (aTC), and reduced activation than the TD group in the left VLPFC.

Conclusion: The observed results might reflect reduced regional activation in ASD and ASD with comorbid depression.

Keywords: autism spectrum disorder, depression comorbidity, functional near-infrared spectroscopy, emotional face recognition, social cognition, frontotemporal activation

\section{Introduction}

University or graduate school students often visit the counselling room for maladaptation to campus life. Several of them have trouble in interpersonal relations with their instructors, peers, and parents due to their autism spectrum disorder (ASD)-related characteristics. ASD is defined as a neurodevelopmental disorder with deficits in social communication and interaction, along with restricted and repetitive patterns of behavior. ${ }^{1}$ Some of the students with ASD have typical depression-associated symptoms such as depressed mood, diminished interest, reduced ability to think or concentrate, feelings of worthlessness, and restricted physical movements. Depression is common among individuals with ASD; social functioning and depression were suggested to be closely intertwined in ASD. ${ }^{2}$ A recent meta-analysis reported increased depression rates in ASD to be associated with increase in age (of the total sample, participants younger than 18 years were 
$<10 \%$ ) and average-to-above average IQ. ${ }^{3}$ Therefore, university and graduate school students may be a particularly vulnerable population for ASD with comorbid depression.

In clinical settings, screening and assessment of depression in ASD individuals is often difficult due to the validity issues with self-report, poor insight (commonly seen in ASD), and overlapping symptoms of ASD and depression such as constricted affect and social withdrawal. ${ }^{4}$ In addition, alexithymia and deficits in emotion regulation are commonly seen in individuals with ASD and in those with mood disorders including depression. ${ }^{5}$ These overlapping symptoms may hinder the clinician's pertinent diagnosis of depression in ASD patients. ${ }^{4}$ Furthermore, because of inability to recognize and label emotions and social communication deficits, they may find it difficult to identify and express their emotional states, and this may lead to depressive symptoms being overlooked by their families and clinicians. ${ }^{6}$

Although medication is not effective in reducing symptoms of $\mathrm{ASD},{ }^{4}$ cognitive behavioral therapy (CBT) is shown to be effective at reducing depression symptoms associated with ASD. ${ }^{7}$ Therefore, appropriate diagnosis of depression in ASD is clinically important. Furthermore, to improve the accuracy of diagnosis, better understanding of the characteristics of ASD with depression, using an objective indicator, may prove to be helpful.

Among neuroimaging tools, functional near-infrared spectroscopy (fNIRS) is a non-invasive optical neuroimaging technique that measures changes in the concentrations of oxygenated [oxy-Hb] and deoxygenated [deoxy- $\mathrm{Hb}$ ] hemoglobin that reflect regional cerebral blood volume and brain activation. ${ }^{8}$ Previous fNIRS studies examining frontotemporal activation during non-social cognition task and verbal fluency task had reported that adult patients with $\mathrm{ASD}^{9}$ and late-onset major depression ${ }^{10}$ showed reduced activation in the bilateral prefrontal cortex compared with healthy controls. In addition, another fNIRS study reported that severity of depression was associated with the integral value of blood flow in the frontal lobe, irrespective of psychiatric diagnosis. ${ }^{11}$ Furthermore, other functional MRI (fMRI) and fNIRS studies using the emotional face recognition task (EFRT) as a social cognition task to activate regional brain function reported that adolescents with major depressive disorder showed lower activation in right insula, superior/middle temporal gyrus, and Heschl's gyrus when viewing fearful faces compared with healthy controls, ${ }^{12}$ and that ASD individuals showed reduced activation in the left prefrontal region compared to those with typical development (TD) during facial expression matching task depicting fear or anger. ${ }^{13}$ Emotion recognition is the ability to identify emotional facial expressions; it is one of the essential components for social cognition, ${ }^{14}$ and deficits in emotion recognition were suggested to be an important feature for understanding ASD. ${ }^{15}$ Thus, we have used an emotional face recognition task for measuring frontotemporal brain function in ASD in this study. To our knowledge, there has been no study that compared brain activity during social cognition tasks among ASD with and without comorbid depression, and TD individuals. The aim of this study was to examine the differences in brain function between ASD young adults with and without comorbid depression and $\mathrm{TD}$, in response to a social cognition task using fNIRS, and to identify specific and distinguishing characteristics of ASD with or without depression that may possibly be utilized as a diagnostic aid. Based on evidences from fMRI and fNIRS studies in ASD and depression, we hypothesized that young adults having ASD with and without depression would show reduced prefrontal brain activation during the social cognition task compared to young adults with TD. Additionally, we also hypothesized that there would be a difference in temporal activation during social cognition tasks between ASD with and without comorbid depression.

\section{Materials and Methods Participants}

Twenty-four young adults with ASD (12 having ASD and comorbid depression [ASD-Dep(+)] and 12 having ASD without depression [ASD-Dep(-)]) and 12 young adults with TD were included. Participants in the ASD-Dep $(+)$ and ASD-Dep(-) groups were diagnosed according to the Diagnostic and Statistical Manual of Mental Disorders, 5th Ed. ${ }^{1}$ Autism Diagnostic Observation Schedule Second Edition, ${ }^{16}$ and Autism Diagnostic Interview-Revised. ${ }^{17}$ In addition, to confirm if there was a current major depressive episode and to screen for current and past comorbid psychiatric disorders, the Mini International Neuropsychiatric Interview ${ }^{18}$ was performed. They had been recruited from among university or graduate school students who visited the mental health counseling room at Chiba University for consultation. The TD subjects were also recruited from among Chiba University students. The three groups, ASD$\operatorname{Dep}(+)$, ASD-Dep(-), and TD, were matched for gender, age, years of education, and intelligence quotient (IQ) measured using the Wechsler Adult Intelligence Scale 
Table I Demographic and Clinical Characteristics of the Subjects

\begin{tabular}{|c|c|c|c|c|c|}
\hline & ASD-Dep(+) & ASD-Dep(-) & TD & \multirow[t]{2}{*}{ p-value ${ }^{a}$} & \multirow[t]{2}{*}{ Tukey's Post Hoc Analysis } \\
\hline & $n=12$ & $n=12$ & $n=12$ & & \\
\hline Age (years) & $22.17(2.25)$ & $22.67(1.56)$ & $21.83(0.94)$ & 0.477 & \\
\hline Gender (male/female) & Male 8/female 4 & Male $9 /$ female 3 & Male $9 /$ female 3 & $0.87 I^{\mathrm{b}}$ & \\
\hline Education (years) & $16.00(2.04)$ & $16.17(1.75)$ & $15.50(0.67)$ & 0.575 & \\
\hline IQ & $119.92(12.01)$ & 118.17 (8.96) & $112.92(8.94)$ & 0.223 & \\
\hline AQ-J & $36.75(3.4 I)$ & $34.42(5.62)$ & - & $0.232^{c}$ & \\
\hline BDI-II & $28.92(9.73)$ & $14.58(5.85)$ & - & $<0.001^{\mathrm{c}}$ & \\
\hline Mean reaction time (ms) & $1060.17(279.78)$ & $1050.17(177.17)$ & $834.75(218.12)$ & 0.035 & ASD-Dep $(+)=$ ASD-Dep $(-)=$ TD \\
\hline Accuracy (\%) & $96.40(5.56)$ & 96.88 (7.77) & $99.48(1.80)$ & 0.362 & \\
\hline
\end{tabular}

Notes: Data are shown as means (SD). ${ }^{\mathrm{a} O n e-w a y ~ a n a l y s i s ~ o f ~ v a r i a n c e, ~}{ }^{\mathrm{b}} \mathrm{Chi}$-square test. Chi square value $=0.277$, 'Student's $t$-test.

Abbreviations: ASD, Autism spectrum disorder; ASD-Dep(+), ASD with comorbid depression; ASD-Dep(-), ASD without depression; TD, typically developed controls; IQ, intelligence quotient; AQ-J, autism quotient Japanese version; BID-II, Beck depression inventory scale 2nd edition.

3rd Edition ${ }^{19}$ (Table 1). The exclusion criteria were lefthandedness, IQ 100 or lower, history of major physical illness and/or head injury, neurological disorder, substance use, and alcohol abuse. None of the participants were under psychotropic medication. Subjective depression severity was assessed by Beck Depression Inventory Scale 2nd edition (BDI-II), ${ }^{20}$ and ASD characteristics were evaluated by the Autistic-Spectrum Quotient Japanese version (AQ-J). ${ }^{21}$ The BDI-II is a self-rating questionnaire that can measure the presence and severity of depression in adults and young adults. It includes 21 questions that assess the somatic, cognitive, and affective symptoms of depression. The question items are scored on four-point scales (the range of the score is 0 to 3 ) with a maximum total score of 63 (higher scores means severer depressive symptoms). ${ }^{20}$ The AQ-J is a self-rating questionnaire that can measure the degree to which an adult with normal intelligence has traits related to the autistic spectrum. $^{21}$ The AQ-J includes 50 questions, and each of the question items is scored 1 point when the respondent records the abnormal or autistic-like behavior with a maximum total score of $50 .^{21}$

This study was conducted in accordance with the Declaration of Helsinki. Participants were explained the content of this study with oral and written information, and written informed consent was obtained before participation. This study was approved by the Research Ethics Committee of Chiba University on the 27th of December, 2017 (approval number: 29-03).

\section{Emotional Face Recognition Task (EFRT)}

An EFRT that was developed to evaluate participants' face perception and their recognition of facial emotions, was used for the fNIRS measurement. The participants were instructed to judge whether the emotion on the face in the presented picture was anger or fear and to respond by pressing a button on a dedicated controller with the thumb of their preferred hand as fast as possible when a particular picture appeared. The baseline task was a gender-matching task (GMT) that involved judging the gender of the presented face and responding by pressing a button, similar to the EFRT. As the GMT was used to cancel sensorimotor artefacts due to finger and body movements from the fNIRS signal change during the EFRT, it was not included in statistical analyses. We referred to previous studies ${ }^{22,23}$ that have utilized emotional face recognition tasks. These studies required the participants to choose one of the two emotion-depicting faces in the lower panel to match the target face picture in the upper panel. Therefore, this process required the participants to not only recognize facial emotions but also to match that emotion to that on another face. Consequently, we tried to make the task simpler and evaluated only the ability of emotional face recognition of the participants and then examined its effects in this study. We used pictures of the facial emotions of "anger" and "fear", since negative emotions such as anger and fear minimize heterogeneity due to valence ${ }^{24}$ and show more robust brain activation compared to positive emotions in patients with neurodevelopmental disorders. ${ }^{25}$ Since gender matching tasks did not require emotional recognition as a response to the task, we set gender matching tasks as neutral control tasks. We hypothesized that when the participants performed this neutral control task, their emotional recognition ability will not be brought into play. We set the frequency of face stimuli presentation at $2 \mathrm{~s}$, with a 1 


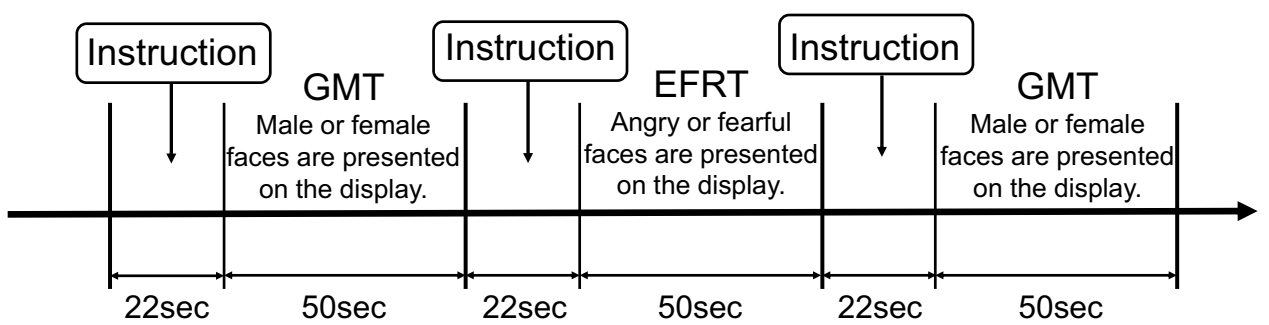

Figure I Designs of the emotional face recognition and gender-matching tasks used in fNIRS measurements.

Notes: Participants were required to respond whether the presented face pictures were male or female in the gender-matching task (GMT), and angry or fearful in the emotional face recognition task (EFRT) by pushing the button on the controller.

$\mathrm{s}$ inter-stimulus interval. There were 16 trials using one block with a total of three blocks, and the GMT were performed before and after the EFRT as a reference (Figure 1). The total duration of one block was $50 \mathrm{~s}$ for the EFRT along with two GMTs (Figure 1). Behavioral performance was assessed by the accuracy (\%) and mean reaction time $(\mathrm{ms})$ for the EFRT (Table 1). The face pictures used were selected from Japanese and Caucasian facial expressions of emotion and neutral faces. ${ }^{26}$ The tasks were administered using Presentation software (https://www.neurobs.com/).

\section{NIRS Measurements}

A 52-channel NIRS machine (ETG-4000, Hitachi Medical Corporation, Tokyo, Japan) was used. Based on the modified Beer-Lambert law, ${ }^{27}$ the relative changes in [oxy-Hb] and [deoxy-Hb] were measured using two infrared light wavelengths (695 and $830 \mathrm{~nm}$ ). The distance between emitter and detector probes was set at $3.0 \mathrm{~cm}$. Channels for fNIRS measurement were defined as each measuring area between pairs of emitter and detector probes. The surface of the cerebral cortex can be measured approximately using fNIRS. ${ }^{28}$ The probes were placed on the participants' frontotemporal area, and the lowest probes were placed along the T4-Fpz-T3 line based on the International 10/20 system. According to the anatomical craniocerebral correction via the International 10/20 system, ${ }^{29}$ this arrangement can measure hemoglobin levels in the bilateral dorsolateral prefrontal cortex (DLPFC), ventrolateral prefrontal cortex (VLPFC), frontopolar cortex, and the anterior part of the superior and middle temporal cortices (aTC). The correspondence between fNIRS channels and measurement points in the cerebral cortex was presented according to the virtual registration method. ${ }^{30}$

The time resolution of the NIRS signal was $0.1 \mathrm{~s}$. We used a moving average window of $5 \mathrm{~s}$ for the analyses to remove any short-term motion artifacts. Moreover, we applied an automated method for rejecting artifacts, focused on three types of noise (high frequency, low frequency, and no signal) and body movement. ${ }^{31}$

We used the EFRT as an activation task and the GMT before and after EFRT for baseline control in this study. To examine task-related activation, linear trend fitting was performed for the data obtained between the two baseline phases (ie, pre- and post-EFRT). The pre- and post-EFRT baselines were defined as the mean values of the 10 -s period immediately prior to the EFRT and at the end of the 10-s period in the GMT following the EFRT.

\section{Statistical Analysis}

Statistical analyses were performed using PASW Statistics (version 27.0; SPSS Japan Inc., Tokyo, Japan). First, we compared the three groups, ASD-Dep(+), ASD-Dep(-), and TD, to confirm that there were no significant differences in age, years of education, and IQ using one-way analyses of variance (ANOVAs), and gender using a chisquare test. In addition, differences in AQ-J and BDI-II scores between ASD-Dep(+) and ASD-Dep(-) were evaluated using Student's $t$-test. Further, to analyze the behavioral performance in EFRT, we compared the mean reaction time and accuracy of the response of the three groups using one-way ANOVA, and post-hoc Tukey's tests $(p<0.05)$ were performed if there were significant differences in mean reaction time or accuracy. We also tested the correlation between clinical variables such as IQ, AQ$\mathrm{J}$, and BDI-II scores and performance parameters using Spearman's rank-order correlation, if there was a significant difference between the ASD groups and TD group. For the fNIRS data, we calculated the mean changes in [oxy-Hb] and [deoxy-Hb] during the EFRT (50 s) in each channel for every participant. We used the inferior 31 channels among the 52 channels for statistical analysis following the methods of previous fNIRS studies $^{31-33}$ and focused on increases in [oxy-Hb] because 
of its superior signal-to-noise ratio. ${ }^{34}$ Moreover, we compared the mean $[\mathrm{oxy}-\mathrm{Hb}]$ changes in the three groups using a one-way ANOVA to analyze differences in frontotemporal function. Mean [oxy-Hb] data were used as the dependent variable, and diagnosis was used as the independent variable. For [oxy-Hb] data, the NIRS signal was expressed as the product of oxy-hemoglobin concentration change and optical path length. The unit of measurement is $\mathrm{mM} \cdot \mathrm{mm}$. Since the optical path length in a participant's brain region cannot be measured, we could not compare channels directly to assess the possibility that the optical path length varies at an individual level. Therefore, we performed 31 one-way ANOVAs for each channel, and the false discovery rate (FDR) approach was adopted to determine the significance level and prevent an increase in alpha error due to the use of multiple comparisons. ${ }^{35}$ Posthoc Tukey's tests $(\mathrm{p}<0.05)$ were performed if significant variables were present. We calculated Spearman's rank correlation coefficients to examine the relationship between $[\mathrm{oxy}-\mathrm{Hb}$ ] changes during the EFRT and demographic or clinical variables, when significant [oxy- $\mathrm{Hb}$ ] differences in the channels were found between the ASD and TD groups. Statistical significance was set at $\mathrm{P}<0.05$.

\section{Results}

Demographic and Clinical Characteristics

The demographic and clinical characteristics of the participants are shown in Table 1. There were no significant differences between ASD-Dep(+), ASD-Dep(-), and TD groups in age, gender, years of education, and IQ (Table 1). On comparing the ASD groups, student's $t$-test showed that the BDIII score of ASD-Dep $(+)$ group was significantly higher than that of ASD-Dep(-) group, although no group difference was observed in the AQ-J score (Table 1).

\section{Behavioral Performance in EFRT}

There was a significant group difference in mean reaction time for EFRT $\left(\mathrm{F}_{2,33}=3.713, \mathrm{p}=0.035\right)$. In the post-hoc analysis, there was no significant difference between the ASD groups and TD group (Table 1). For the accuracy of the response in EFRT, there was no significant difference across the three groups $\left(\mathrm{F}_{2,33}=1.049, \mathrm{p}=0.362\right)$ (Table 1).

\section{Hemodynamic Response During the EFRT} The grand-mean [oxy-Hb] waveforms during EFRT in each group are shown in Figure 2. One-way ANOVA revealed significant differences between the ASD groups

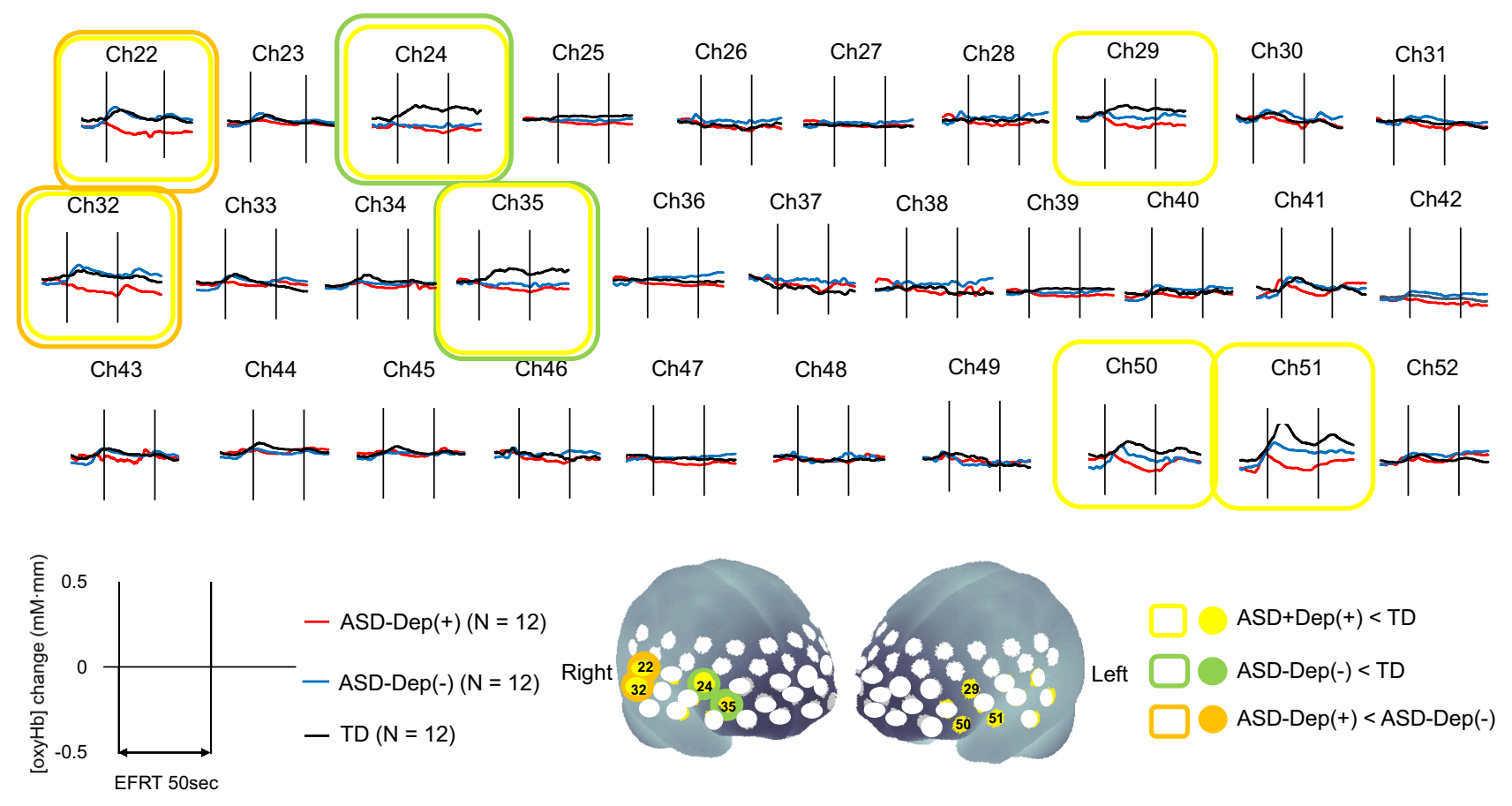

Figure 2 The grand-averaged waveforms of oxygenated hemoglobin concentration ([oxy-Hb]) changes.

Notes: The images in the upper part of the figure show the grand-averaged waveforms of [oxy-Hb] changes during the emotional face recognition task (EFRT) in autism spectrum disorder (ASD) with comorbid depression (red), ASD without depression (blue), and typical development (TD) (black) participants. In the brain mapping images in the lower part of the figure, colored circles indicate the significant channels in one-way ANOVA (false discovery rate-corrected $p<0.05$ ). In addition, the results of multiple comparisons are shown as follows: yellow circle, ASD with depression < TD; green circle, ASD without depression < TD; orange circle, ASD with depression < ASD without depression. 
and TD group in seven channels (CHs 22, 24, 29, 32, 35 , 50, and 51) located approximately in the bilateral VLPFC and right aTC (FDR-corrected $\mathrm{p}<0.05)$. In the multiple comparisons of the seven significant channels, the mean [oxy-Hb] changes in the ASD-Dep(+) group were significantly smaller than those of the TD group in all of the seven channels. The mean oxy-Hb changes in ASD-Dep(-) group were significantly smaller than those of the TD group in two channels (CHs 24 and 35). Compared with TD group, reduced activation was more commonly observed in $\mathrm{CHs} 24$ and 35, which are located approximately in the right VLPFC, in both of the ASD-Dep(+) and ASD-Dep(-) groups. In direct comparison between ASD-Dep $(+)$ and ASD-Dep(-) groups, the mean [oxy-Hb] changes in the ASD-Dep $(+)$ group were significantly smaller than those in ASDDep(-) group in CHs 22 and 32, which is located approximately in the right aTC.

\section{Correlation Between NIRS Signals and Demographic/Clinical Characteristics and EFRT Performance in ASD}

We found no significant correlations between demographic/clinical variables (ie, age, years of education, IQ, AQ-J, and BDI-II scores) or EFRT performance (mean reaction time and accuracy of response) and mean [oxy$\mathrm{Hb}$ ] changes during EFRT in participants with ASD in CHs 24 and 35 or in participants with $\operatorname{ASD}-\operatorname{Dep}(+)$ in CHs 22, 29, 32, 50 and 51.

\section{Discussion}

We examined the emotional recognition characteristics in ASD with and without depression using an emotional face recognition task. However, there are various studies which have examined emotion recognition in ASD and depression. For example, previous studies have examined the emotional recognition in ASD participants by voice ${ }^{36}$ and gestures $^{37}$ using video analysis and reported characteristics in ASD that can help improve the accuracy of the diagnosis or assessment of ASD. Additionally, emotion recognition deficits in major depression were found by matching the emotional face stimuli to a semantic label. ${ }^{38}$ Nevertheless, to the best of our knowledge, this is the first study comparing frontotemporal activity during a social cognition task such as EFRT between young adults with ASD-Dep(+) and ASD-Dep(-) using fNIRS. Comparison of frontotemporal activation between the two
ASD groups and the TD group revealed that both ASD groups exhibited reduced activation of right VLPFC during a social cognition task. Specifically, among the two ASD groups, the ASD-Dep(+) group showed reduced activation in the right aTC during the social cognition task compared to the ASD-Dep(-) group, suggesting that there might be some clues in the right aTC that points towards specific characteristics of depression in ASD young adults. In addition, the ASD-Dep(+) group showed reduced activation during the social cognition task in the left VLPFC compared to the TD group, although the ASD-Dep(-) group did not show significant difference compared to the ASD-Dep $(+)$ or TD groups. No association was observed between age, years of education, IQ, or task performance and the right VLPFC or aTC activation during the EFRT in ASD individuals, showing that the effects of these factors on the right VLPFC or aTC activity were not significant. These findings indicate the involvement of the right VLPFC in the social cognition deficits in ASD and the left VLPFC in ASD with comorbid depression, and that analyzing the right aTC might help distinguish ASD young adults with comorbid depression from those without the comorbidity.

\section{Prefrontal Activity in Young Adults with ASD}

An fMRI study that examined brain function during the EFRT reported reduced left inferior frontal activation in ASD compared with TD. ${ }^{12}$ In addition, an fNIRS study reported reduced brain activation in response to the EFRT in left brain areas corresponding to the inferior and middle frontal gyri and anterior superior and middle temporal gyri demonstrating the association of these areas with difficulties in social cognition skills in ASD. ${ }^{22}$ In this study, ASD groups showed reduced activation during EFRT in the right VLPFC compared to TD group, although the ASD$\operatorname{Dep}(+)$ group showed reduced activation in the left VLPFC. The reason for the divergence of the present study results from previous functional imaging studies cannot be revealed solely from the study. However, the background of the participants may have partially affected the observed divergence. The participants were university or graduate school students with relatively high IQ and education. Higher intelligence is associated with better social cognition in ASD. ${ }^{39}$ Therefore, the ASD individuals with relatively high intelligence and education without the effect of comorbid depression in this study might have had 
better social cognition than the participants of previous studies, and thereby, the effect of social cognition deficits on the left VLPFC activation during the social cognition tasks might not have reached statistical significance. Moreover, the differences in the EFRT content might also have affected the divergence in the results. We simplified the EFRT content that was used in the previous fNIRS studies ${ }^{22,23}$ to focus on the simple response to fear or anger on the face depicted. A further study that reveals the effects of intelligence on the left VLPFC activation during social cognition tasks in ASD and the effects of differences in the EFRT content is expected and would answer for this divergence.

Considering another aspect of the participants, ASDDep(+) and ASD-Dep(-) groups had difficulties in adapting to their campus or daily life due to ASD characteristics, and had sought counseling for their problems in relationships with instructors, peers, or parents. They may experience social pain in their lives. Social pain refers to a painful experience associated with damage to desired social connections, ${ }^{40}$ and previous studies have demonstrated that the right VLPFC is involved in the downregulation of social pain. ${ }^{41,42}$ The observed reduced activation of right VLPFC during the social cognition task might be related to deficits in the downregulation of social pain and cause problems that make ASD young adults require consultation in the university counseling room. Furthermore, on the structure and function of the right VLPFC, previous studies have reported reduced gray matter volume in the $\mathrm{IFG}^{43,44}$ and reduced neural activity in the temporo-parietal junction, right IFG, and left premotor cortex during social tasks ${ }^{45}$ in ASD compared with TD participants. Further studies with larger sample sizes, including ASD subgroups with diverse backgrounds, are expected to confirm the role of the right VLPFC in delineating characteristics of ASD.

\section{Right aTC Activity and ASD Young Adults with Depression}

The ASD-Dep $(+)$ group showed a significantly reduced activation during the EFRT in the right aTC compared with the ASD-Dep(-) and TD groups. The superior and middle temporal gyri are responsible for processing emotional facial expressions. ${ }^{46}$ Alexithymia and emotion regulation deficits were suggested to be seen in individuals with ASD and mood disorders. ${ }^{5}$ Alexithymia is associated with deficits in labelling emotional facial expressions among clinical disorders, ${ }^{47}$ and the right temporal cortex is involved in cognitive aspects of alexithymia. ${ }^{48}$ A previous fMRI study found that adolescents with major depressive disorder showed lower activation during EFRT in right insula, superior/middle temporal gyrus, and Heschl's gyrus, ${ }^{12}$ while another fMRI study suggested that ASD-related hypoactivation during the emotional-face processing is found in the hypothalamus and not in the aTC area. ${ }^{49}$ In addition, as we described above, previous fNIRS study reported that adult ASD individuals showed reduced activation during EFRT compared with healthy participants not in the right temporal area but the left frontotemporal area. ${ }^{22}$ In the present study, no significant difference was observed in the right aTC activation during the EFRT on comparing ASD-Dep(-) and TD groups, and this was consistent with the aforementioned studies. The specific characteristics of the ASD-Dep(+) and ASD-Dep (-) groups and the reason for the observed divergence in the right aTC activation during the EFRT could not be revealed solely from the findings of this study. However, we speculate that the reduced aTC activation observed during EFRT in the ASD-Dep(+) group might reflect the reduced temporal activation associated with depression rather than ASD. Moreover, since there was a significant difference in the aTC activation during EFRT between ASD-Dep(+) and ASD-Dep(-) groups, the activation in the right aTC during the EFRT could be a clue to discriminate individuals with ASD with and without comorbid depression. Further study on the differences between ASD with and without comorbid depression focusing on aTC function and its role in the neural systems might explain the mechanism that brought about the observed reduction in right aTC activation during EFRT in the ASD-Dep $(+)$ young adults.

\section{Limitations}

Several limitations should be considered in the interpretation of the observed results. First, fNIRS measures brain activity only on the brain surface. Hence, we could not measure activity in deep brain structures such as the limbic system. Second, we could not examine the neural and functional connectivity between prefrontal or temporal and other brain regions. Further studies examining them might reveal the mechanisms that led to the divergent temporal activation between ASD-Dep $(+)$ and ASD-Dep (-) groups during social cognition tasks. Third, we did not measure heart rates or electrocardiogram simultaneously with fNIRS signals in this study. Therefore, changes in 
[oxy-Hb] and [deoxy-Hb] that were not due to functional brain activity could not be controlled, and systemic or physiological effects on the observed results could not be excluded in this study. Further study that examines these effects on $[0 x y-H b]$ and [deoxy-Hb] in the same study design would be necessary to confirm the validity of the observed results. Fourth, we did not perform a structured interview such as the Hamilton Rating Scale for Depression in order to evaluate depression severity, but used a self-rating questionnaire (ie BDI-II). Thus, it would be necessary to consider the validity of the subjective data in the interpretation for the observed correlations between the right VLPFC activation and depression severity. Further studies using objective assessment tools would be required to confirm the validity of the present results. Fifth, the participants consisted of only university or graduate school students. Therefore, the findings cannot be generalized to a broader population of ASD individuals based on the current study. Sixth, the sample size was small, and this may limit the demographical variability and may affect the statistical results. Thus, further study, replicated with a larger sample size is required to confirm the validity of the present results. Finally, this study did not examine typically developed individuals with depression. Including this group in the analysis would provide additional findings to understand the characteristics of ASD-Dep $(+)$ group and may be considered in the future.

\section{Conclusion}

Using fNIRS, we found differences in frontotemporal hemodynamic responses associated with social cognitive activation between ASD with and without comorbid depression, and TD groups. The right VLPFC showed reduced activation in both the ASD groups (ie, ASD-Dep(+) and ASD-Dep(-) groups) compared with TD group. Furthermore, reduced activation in the right aTC might indicate comorbid depression in young adults with ASD. To our knowledge, this is the first study to compare regional brain activation during social cognition task in young adults with ASD and those with comorbid depression. The reason for the observed reduced activation in the right aTC during EFRT in ASD with the comorbid depression group could not be explained solely by this study. However, the observed findings in this study can give a clue to understanding the characteristics of ASD with depression.

\section{Acknowledgments}

This study was supported by the Japan Society for the Promotion of Science, KAKENHI (Grant-in-Aid for
Scientific Research (C), 20K03434, to TO). We appreciate the participants of this study. In addition, we would like to thank Editage for English language editing.

\section{Disclosure}

ES reports lecture fees from Mochida Pharmaceutical, Yoshitomiyakuhin Corporation, Kyowa pharmaceutical, Eisai, and Sumitomo Dainippon Pharma, and a joint research fund under contract from Sumitomo Dainippon Pharma, outside the submitted work. The authors report no other conflicts of interest in this work.

\section{References}

1. American Psychiatric Association. Diagnostic and Statistical Manual of Mental Disorders. 5th ed. Washington. DC:American Psychiatric Association; 2013

2. Schiltz HK, McVey AJ, Dolan BK, et al. Changes in depressive symptoms among adolescents with ASD completing the PEERS ${ }^{\circledR}$ social skills intervention. J Autism Dev Disord. 2018;48(3):834-843. doi:10.1007/s10803-017-3396-6

3. Hudson CC, Hall L, Harkness KL. Prevalence of depressive disorders in individuals with autism spectrum disorder: a meta-analysis. J Abnorm Child Psychol. 2019;47(1):165-175. doi:10.1007/s10802018-0402-1

4. Pezzimenti F, Han G, Vasa R, et al. Depression in youth with autism spectrum disorder. Child Adolesc Psychiatr Clin N Am. 2019;28 (3):397-409. doi:10.1016/j.chc.2019.02.009

5. Morie KP, Jackson S, Zhai ZW, Potenza MN, Dritschel B. Mood disorders in high-functioning autism: the importance of alexithymia and emotional regulation. J Autism Dev Disord. 2019;49(7):29 35-2945. doi:10.1007/s10803-019-04020-1

6. Bird G, Cook R. Mixed emotions: the contribution of alexithymia to the emotional symptoms of autism. Transl Psychiatry. 2013;3(7): e285. doi:10.1038/tp.2013.61

7. Chandrassekhar T, Sikich L. Challenges in the diagnosis and treatment of depression in autism spectrum disorders across the lifespan. Dialogues Clin Neurosci. 2015;17(2):219-227.

8. Villringer A, Planck J, Hock C, et al. Near infrared spectroscopy (NIRS): a new tool to study hemodynamic changes during activation of brain function in human adults. Neurosci Lett. 1993;154(12):101-104. doi:10.1016/0304-3940(93)90181-J

9. Kawakubo Y, Kuwabara H, Watanabe K, et al. Impaired prefrontal hemodynamic maturation in autism and unaffected siblings. PLoS One. 2009;4(9):e6881. doi:10.1371/journal.pone.0006881

10. Pu S, Matsumura H, Yamada T, et al. Reduced frontopolar activation during verbal fluency task associated with poor social functioning in late-onset major depression: multi- channel near-infrared spectroscopy study. Psychiatry Clin Neurosci. 2008;62(6):728-737. doi:10.1111/j.1440-1819.2008.01882.x

11. Kawano M, Kanazawa T, Kikuyama H, et al. Correlation between frontal lobe oxy-hemoglobin and severity of depression assessed using near-infrared spectroscopy. J Affect Disord. 2016;205: 154-158. doi:10.1016/j.jad.2016.07.013

12. Hall LMJ, Klimes-Dougan B, Hunt RH, et al. An fMRI study of emotional face processing in adolescent major depression. $J$ Affect Disord. 2014;168:44-50. doi:10.1016/j.jad.2014.06.037

13. Kleinhans NM, Richards T, Weaver K, et al. Association between amygdala response to emotional faces and social anxiety in autism spectrum disorders. Neuropsychologia. 2010;48(12):3665-3670. doi:10.1016/j.neuropsychologia.2010.07.022 
14. Bänziger T, Grandjean D, Scherer KR. Emotion recognition from expressions in face, voice, and body: the Multimodal Emotion Recognition Test (MERT). Emotion. 2009;9(5):691-704. doi:10.10 37/a0017088

15. Harms MB, Martin A, Wallace GL. Facial emotion recognition in autism spectrum disorders: a review of behavioral and neuroimaging studies. Neuropsychol Rev. 2010;20(3):290-322. doi:10.1007/s11065010-9138-6

16. Lord C, Rutter M, DiLavore PC, et al. Autism Diagnostic Observation Schedule. 2nd ed. Torrence, CA:Western Psychological Services; 2012:1-4. (ADOS-2) Manual (Part I) Modules.

17. Le Couteur A, Lord C, Rutter M. Autism Diagnostic InterviewRevised (ADI-R). Los Angeles, CA: Autism Genetic Resource Exchange; 2003.

18. Sheehan DV, Lecrubier Y, Sheehan KH, et al. The Mini-International Neuropsychiatric Interview (M.I.N.I.): the development and validation of a structured diagnostic psychiatric interview for DSM-IV and ICD-10. J Clin Psychiatry. 1998;59(Suppl 20):22-33; quiz 34-57.

19. Wechsler D. Manual for the Wechsler Adult Intelligence Scale. 3rd ed. USA: Psychological Corporation; 1997.

20. Beck AT, Steer RA, Brown GK. Manual for the Beck Depression Inventory-II. San Antonio, TX: Psychological Corporation; 1996.

21. Wakabayashi A, Baron-Cohen S, Wheelwright S, et al. The Autism-Spectrum Quotient (AQ) in Japan: a cross-cultural comparison. J Autism Dev Disord. 2006;36(2):263-270. doi:10. 1007/s10803-005-0061-2

22. Hirata K, Egashira K, Harada K, et al. Differences in frontotemporal dysfunction during social and nonsocial cognition tasks between patients with autism spectrum disorder and schizophrenia. Sci Rep. 2018;8(1):3014. doi:10.1038/s41598-018-21379-w

23. Watanuki T, Matsuo K, Egashira K, et al. Precentral and inferior prefrontal hypoactivation during facial emotion recognition in patients with schizophrenia: a functional near-infrared spectroscopy study. Schizophr Res. 2016;170(1):109-114. doi:10.1016/j.schres.20 15.11.012

24. Sugranyes G, Kyriakopoulos M, Corrigall R, Taylor E, Frangou S. Autism spectrum disorders and schizophrenia: meta-analysis of the neural correlates of social cognition. PLoS One. 2011;6(10):e25322. doi:10.1371/journal.pone. 0025322

25. Bora E, Pantelis C. Meta-analysis of social cognition in attention-deficit/hyperactivity disorder (ADHD): comparison with healthy controls and autistic spectrum disorder. Psychol Med. 2016;46(4):699-716. doi:10.1017/S0033291715002573

26. Matsumoto D, Ekman P. Japanese and Caucasian Facial Expressions of Emotion (JACFEE) and Neutral Faces (Jacneuf). San Francisco, CA: Intercultural and Emotion Research Laboratory, Department of Psychology, San Francisco State University; 1988.

27. Yamashita Y, Maki A, Ito Y, et al. Noninvasive near infrared topography of human brain activity using intensity modulation spectroscopy. Opt Eng. 1996;35(4):1046-1049. doi:10.1117/1.600721

28. Okada E, Delpy DT. Near-infrared light propagation in an adult head model. II. Effect of superficial tissue thickness on the sensitivity of the near-infrared spectroscopy signal. Appl Opt. 2003;42(16):29 15-2922. doi:10.1364/AO.42.002915

29. Okamoto M, Dan H, Sakamoto K, et al. Three-dimensional probabilistic anatomical cranio-cerebral correlation via the international 10 20 system oriented for transcranial functional brain mapping. NeuroImage. 2004;21(1):99-111. doi:10.1016/j.neuroimage.2003.08. 026

30. Tsuzuki D, Jurcak V, Singh AK, et al. Virtual spatial registration of stand-alone fNIRS data to MNI space. NeuroImage. 2007;34 (4):1506-1518. doi:10.1016/j.neuroimage.2006.10.043

31. Takizawa R, Kasai K, Kawakubo Y, et al. Reduced frontopolar activation during verbal fluency task in schizophrenia: a multi-channel near-infrared spectroscopy study. Schizophr Res. 2008;99(1-3):250-262. doi:10.1016/j.schres.2007.10.025
32. Takei Y, Suda M, Aoyama Y, et al. Temporal lobe and inferior frontal gyrus dysfunction in patients with schizophrenia during face-to-face conversation: a near-infrared spectroscopy study. J Psychiatr Res. 2013;47(11):1581-1589. doi:10.1016/j.jpsychires.2013.07.029

33. Ohtani T, Nishimura Y, Takahashi K, et al. Association between longitudinal changes in prefrontal hemodynamic responses and social adaptation in patients with bipolar disorder and major depressive disorder. $J$ Affect Disord. 2015;176:78-86. doi:10.1016/j.jad.2015.01.042

34. Huppert TJ, Hoge RD, Diamond SG, et al. A temporal comparison of BOLD, ASL, and NIRS hemodynamic responses to motor stimuli in adult humans. NeuroImage. 2006;29(2):368-382. doi:10.1016/j. neuroimage.2005.08.065

35. Singh AK, Dan I. Exploring the false discovery rate in multichannel NIRS. Neuroimage. 2006;33(2):542-549. doi:10.1016/j.neuroimage.2006.06. 047

36. Rutherford M, Baron-Cohen S, Stone V, Wheelwright S. Reading the mind in the voice: a study with normal adults and adults with Asperger syndrome or high functioning autism. J Autism Dev Disord. 2002;32(3):189-194. doi:10.1023/A:1015497629971

37. Rynkiewicz A, Schuller B, Marchi B, et al. An investigation of the "female camouflage effect" in autism using a computerized ADOS-2 and a test of sex/gender differences. Mol Autism. 2016;7(1):10. doi:10.1186/s13229-016-0073-0

38. van Wingen GA, van Eijndhoven P, Tendolkar I, Buitelaar J, Verkes RJ, Fernández G. Neural basis of emotion recognition deficits in first-episode major depression. Psychol Med. 2011;41(7): 1397-1405. doi:10.1017/S0033291710002084

39. Hirosawa T, Kontani K, Fukai M, et al. Different associations between intelligence and social cognition in children with and without autism spectrum disorders. PLoS One. 2020;15(8):e235380. doi:10.1371/journal.pone. 0235380

40. Eisenberger NI. Social pain and the brain: controversies, questions, and where to go from here. Annu Rev Psychol. 2015;66(1):601-629. doi:10.1146/annurev-psych-010213-115146

41. He Z, Liu Z, Zhao J, et al. Improving emotion regulation of social exclusion in depression-prone individuals: a tDCS study targeting right VLPFC. Psychol Med. 2020;50(16):2768-2779. doi:10.1017/ S0033291719002915

42. He ZH, Zhao J, Shen JS, et al. The right VLPFC and down regulation of social pain: a TMS study. Hum Brain Mapp. 2020;41 (5):1362-1371. doi:10.1002/hbm.24881

43. Kosaka H, Omori M, Munesue T, et al. Smaller insula and inferior frontal volumes in young adults with pervasive developmental disorders. Neuroimage. 2010;50(4):1357-1363. doi:10.1016/j.neuroimage.2010. 01.085

44. Yamasaki S, Yamasue H, Abe O, et al. Reduced gray matter volume of pars opercularis is associated with impaired social communication in high-functioning autism spectrum disorders. Biol Psychiatry. 2010;68(12):1141-1147. doi:10.1016/j.biopsych.2010.07.012

45. Kana RK, Libero LE, Hu CP, et al. Functional brain networks and white matter underlying theory-of-mind in autism. Soc Cogn Affect Neurosci. 2014;9(1):98-105. doi:10.1093/scan/nss106

46. Haxby JV, Hoffman EA, Gobbini MI. The distributed human neural system for face perception. Trends Cogn Sci. 2000;4(6):223-233. doi:10.1016/S1364-6613(00)01482-0

47. Grynberg D, Chang B, Corneille O, et al. Alexithymia and the processing of emotional facial expressions (EFEs): systematic review, unanswered questions and further perspectives. PLoS One. 2012;7(8): e42429. doi:10.1371/journal.pone.0042429

48. Bermond B, Vorst HCM, Moormann PP. Cognitive neuropsychology of alexithymia: implications for personality typology. Cogn Neuropsychiatry. 2006;11(3):332-360. doi:10.1080/13546800500368607

49. Aoki Y, Cortese S, Tansella M. Neural bases of atypical emotional face processing in autism: a meta-analysis of fMRI studies. World J Biol Psychiatry. 2015;16(5):291-300. doi:10.3109/15622975.2014. 957719 


\section{Publish your work in this journal}

Neuropsychiatric Disease and Treatment is an international, peerreviewed journal of clinical therapeutics and pharmacology focusing on concise rapid reporting of clinical or pre-clinical studies on a range of neuropsychiatric and neurological disorders. This journal is indexed on PubMed Central, the 'PsycINFO' database and CAS, and is the official journal of The International Neuropsychiatric Association (INA). The manuscript management system is completely online and includes a very quick and fair peer-review system, which is all easy to use. Visit http://www.dovepress.com/testimonials.php to read real quotes from published authors.

Submit your manuscript here: https://www.dovepress.com/neuropsychiatric-disease-and-treatment-journal 